Supplement of Saf. Nucl. Waste Disposal, 1, 13-14, 2021

https://doi.org/10.5194/sand-1-13-2021-supplement

(C) Author(s) 2021. CC BY 4.0 License.

Supplement of

\title{
Structural integrity investigations of spent nuclear fuel with finite element modeling
}

Efstathios Vlassopoulos et al.

Correspondence to: Efstathios Vlassopoulos (vse@nagra.ch)

The copyright of individual parts of the supplement might differ from the article licence. 


\section{Structural Integrity Investigations of Spent Nuclear Fuel with Finite Element Modeling}

S. Pudollek

Interdisciplinary research symposium: On the safety of nuclear disposal practices, Berlin, 10-12 November 2021 


\section{Introduction}

- Swiss waste management includes various back-end activities before SNF final disposal

- wet/dry interim storage, transport, handling and re-packaging for final disposal

- SNF is subjected to various temperature and loading conditions

- Pre-disposal requirements:

- ensure safety of SNF handling and encapsulation operations

- investigate fuel ageing mechanisms during long-term dry interim storage

Swiss waste management concept: SNF flow to final disposal

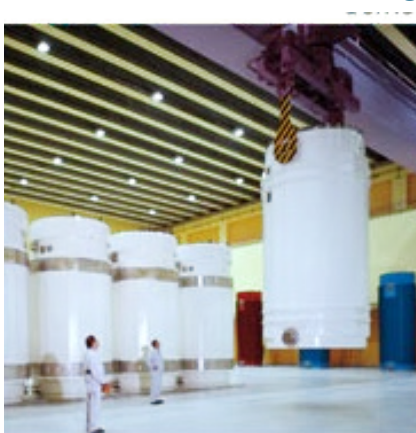

Dry Interim Storage

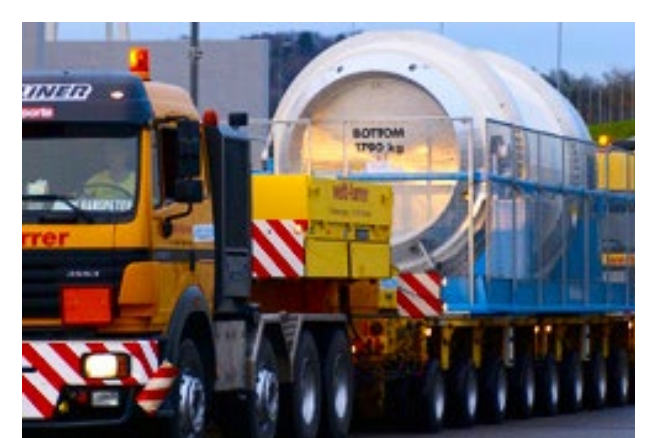

Cask Transport

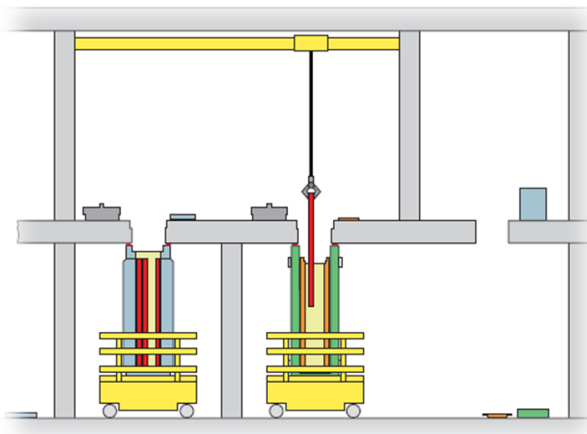

Encapsulation Plant

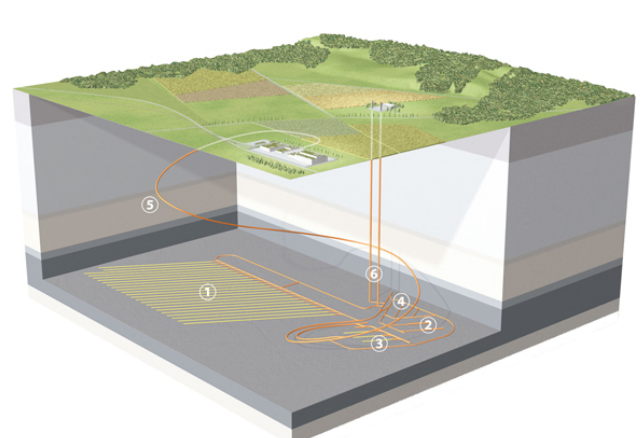

Deep geological Repository

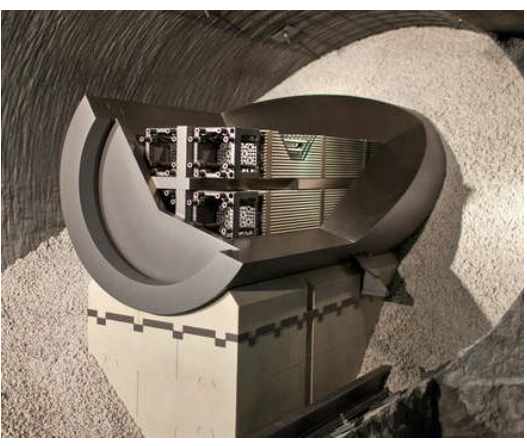

Final Disposal Canister 
- Mechanical response of SNF depends on:

- cladding properties being of highest importance and

- fuel/cladding interaction and composite SNF material properties

- The mechanical properties of nuclear fuel rods change:

- During irradiation (pellet cracking, cladding embrittlement, PCMI, etc.)

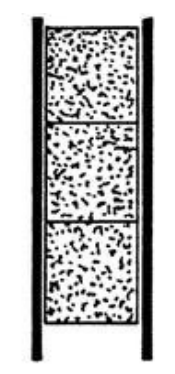

- After irradiation (hydride formation and/or re-orientation, radioactive decay damage, etc.)

- Challenge...

- Predicting mechanical response of SNF in different loading types

- Evaluate consequences in case of SNF rod integrity loss
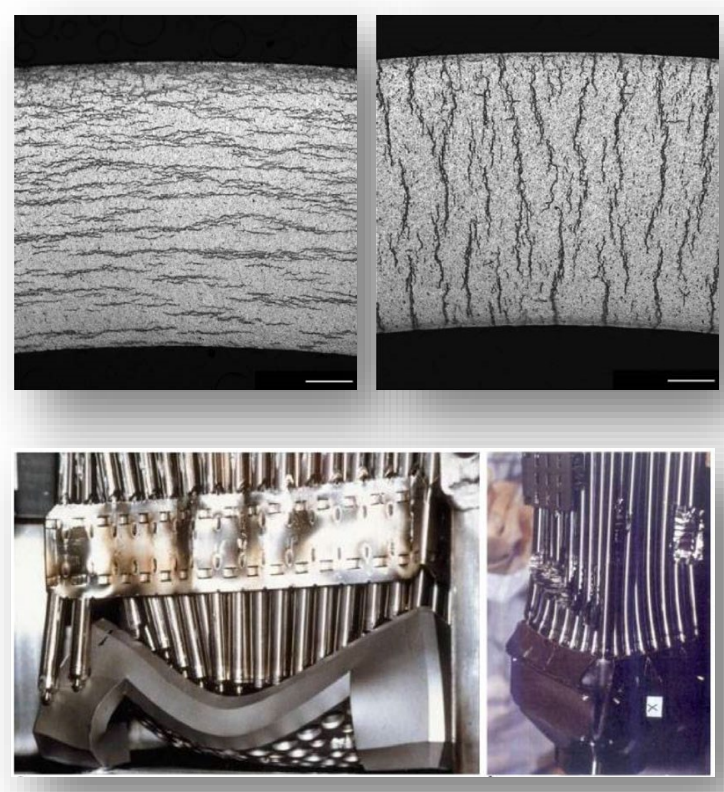


\section{Nagra - SNF Integrity Investigations}

\section{- Objective:}

- Assess the mechanical response of SNF rods in quasi-static \& dynamic loads

- Approach, i.e. experimental / numerical :

- Investigate mechanical properties of SNF rod: empirical laws: $\sigma_{f}=f(B U, \varepsilon)$

- Study rod failure processes and consequences

- Develop Finite Element Models (FEM) validated against experimental data

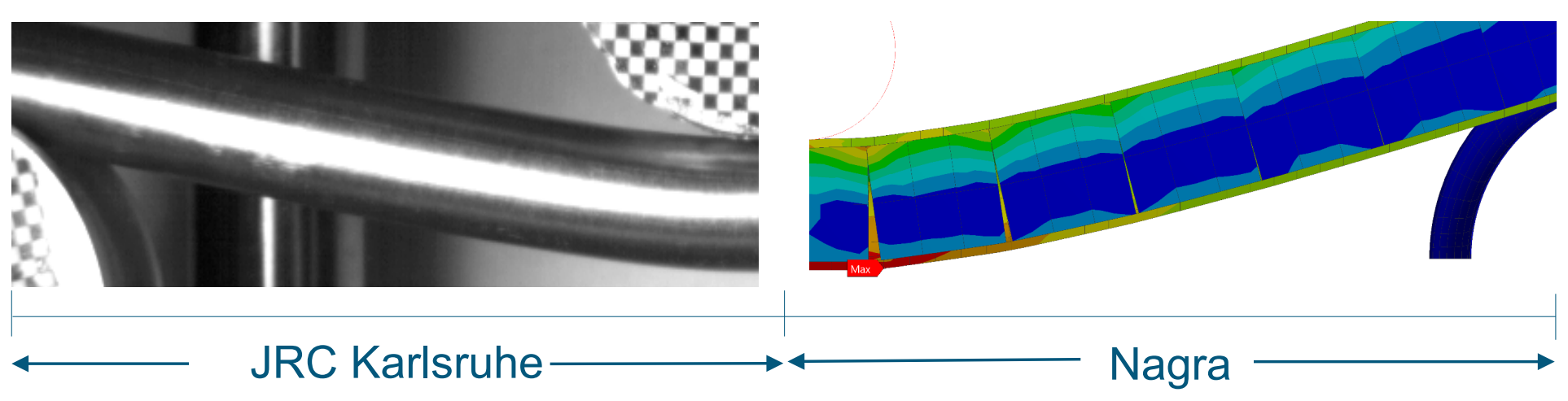




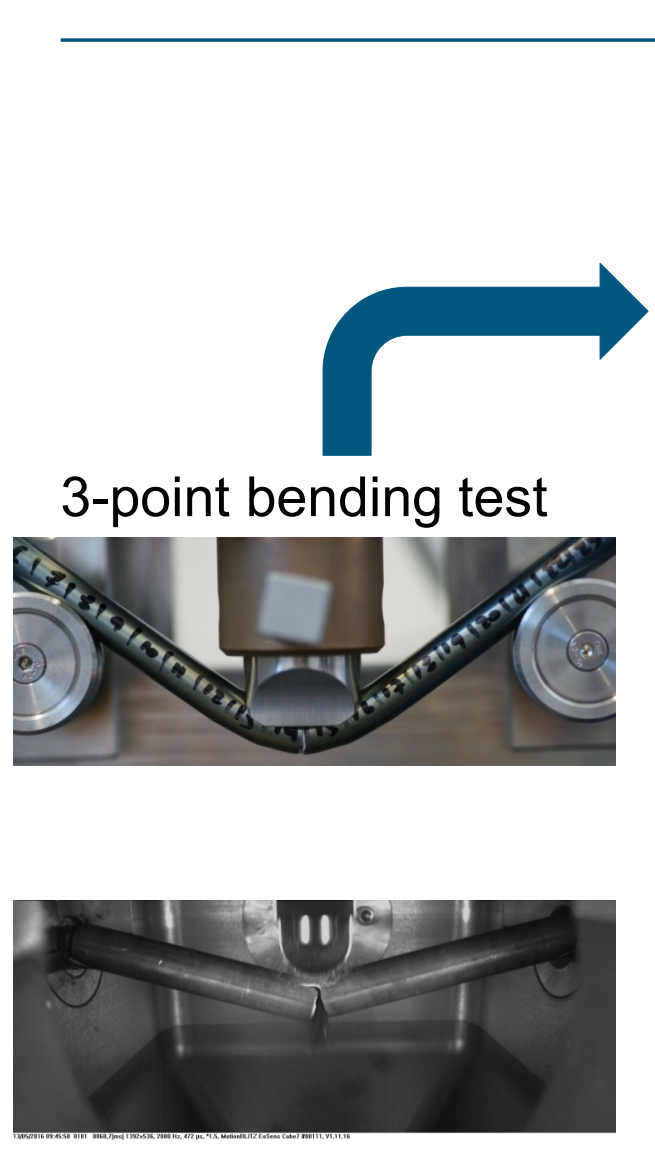

Impact test

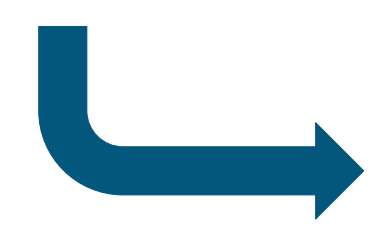

\section{Post-test examinations:}

1. $\mathrm{H}_{2}$ measurement

2. Metallography

3. Fuel mass release

4. Particle size distribution

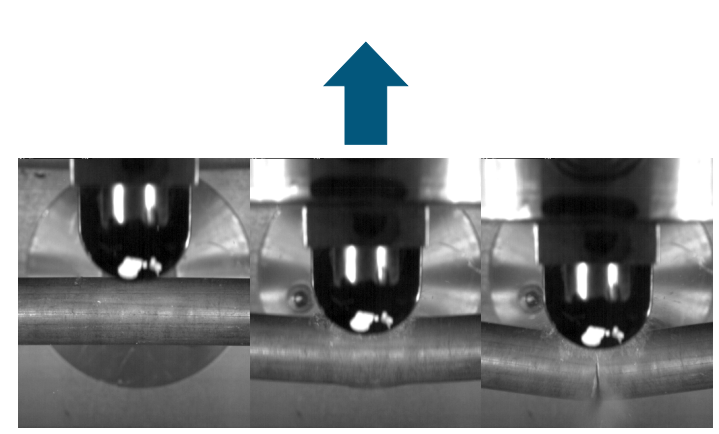

Impact sequence

$$
\sigma_{f}=f(B U, \varepsilon)
$$

3D FEM

Effective mechanical properties

Pellet/Cladding
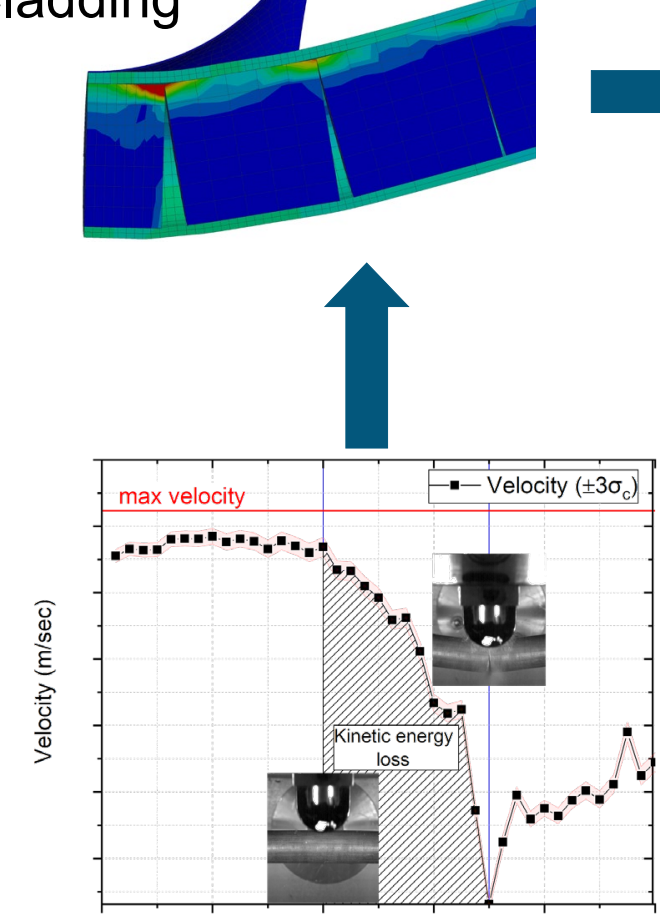

1D FEM

1. SFA integrity investigations

2. Failure criteria 


\section{3-Point Bending Tests - Setup and Method}

- Quasi-static loads: $1 \mathrm{~mm} / \mathrm{min}$ applied with step-motor

- Sensors: pressure, displacement and applied loads

- Use of simple beam theory for flexural properties derivation

- Properties derived as function of:

- Burnup

- Cladding hydrogen content
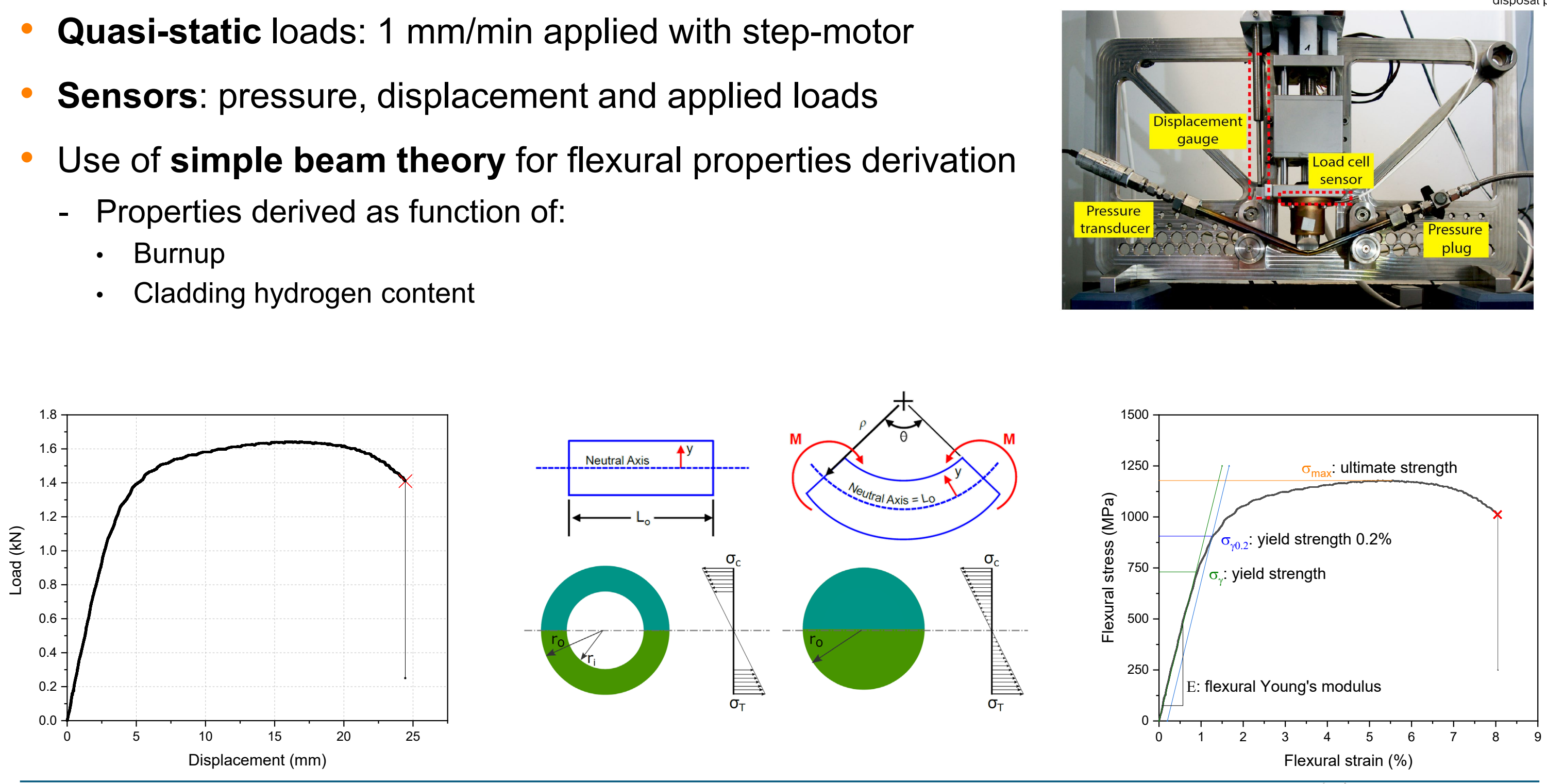


\section{Numerical Investigations of SNF Mechanical Properties}

- Objective:

- Derive effective mechanical properties of SNF rod that reflect the mechanical response of the composite spent fuel/cladding system

- Finite Element Modeling (FEM) in ANSYS Mechanical with implicit static structural analysis for the 3-point bending case

- Modeling approach:

1. Explicit modeling of fuel and pellets (3D FEM) to simulate experimental tests

2. Sensitivity analysis of the model and calibration against experimental results

3. Simplification of modeling approach (1D FEM)

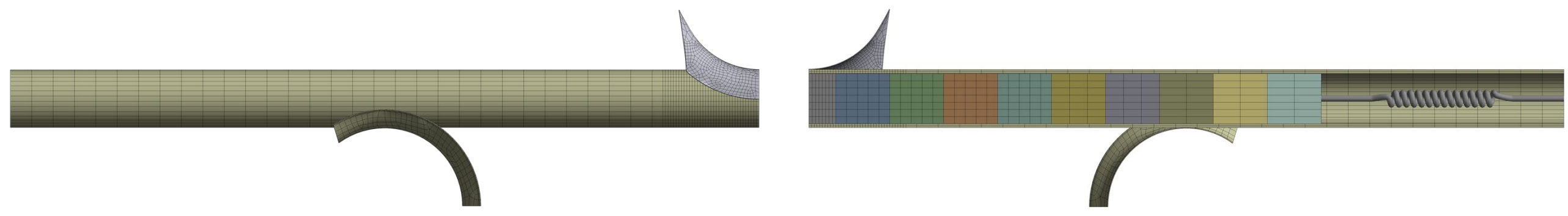


- Development approach:

- Based on 3-point bending tests on surrogate rods

- Uncertainties elimination (known mechanical properties \& fixed geometry)

- Sensitivity analysis:

1. Material model for pellets/cladding

2. Numerical parameters

3. Physical parameters

Voce plasticity model used for cladding

- Final model as:

- Best compromise between solution quality and computational time

\section{- SNF rod effective properties derivation}

- Optimization process minimizing difference between experimental and numerical results

- Evaluate parameters relative importance

- Best model provides material parameters
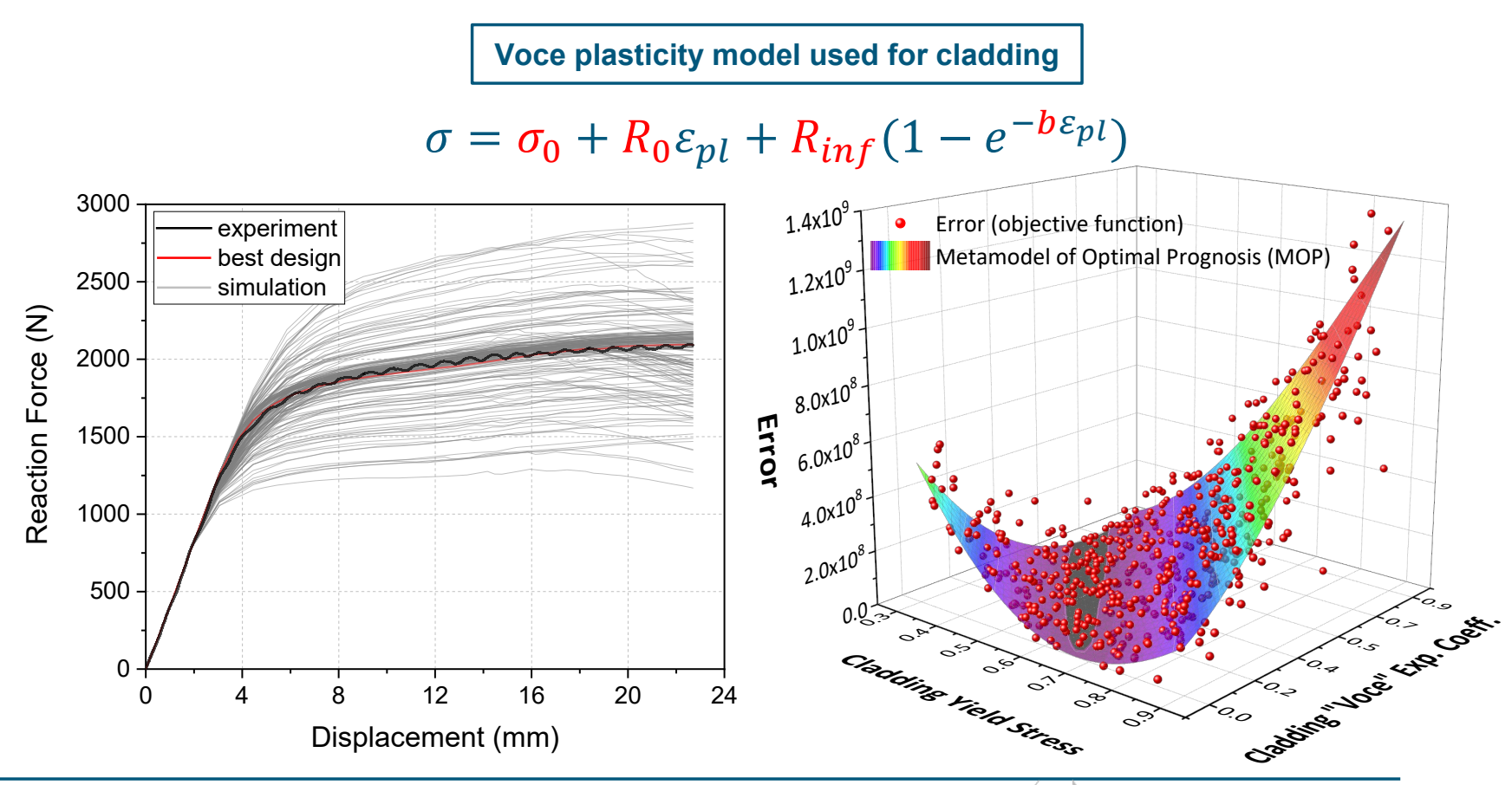


\section{Towards 1D Beam Modeling}

- 3D FE model can accurately predict bending behavior

- However, too slow to be used for a large scale SFA design

\section{EPFL}

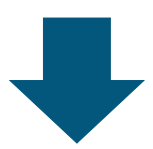

- A 1D beam model was created and optimized to fit experimental data

- Faster convergence compared to the 3D model

- Optimized to predict bending behavior as accurately as the 3D model

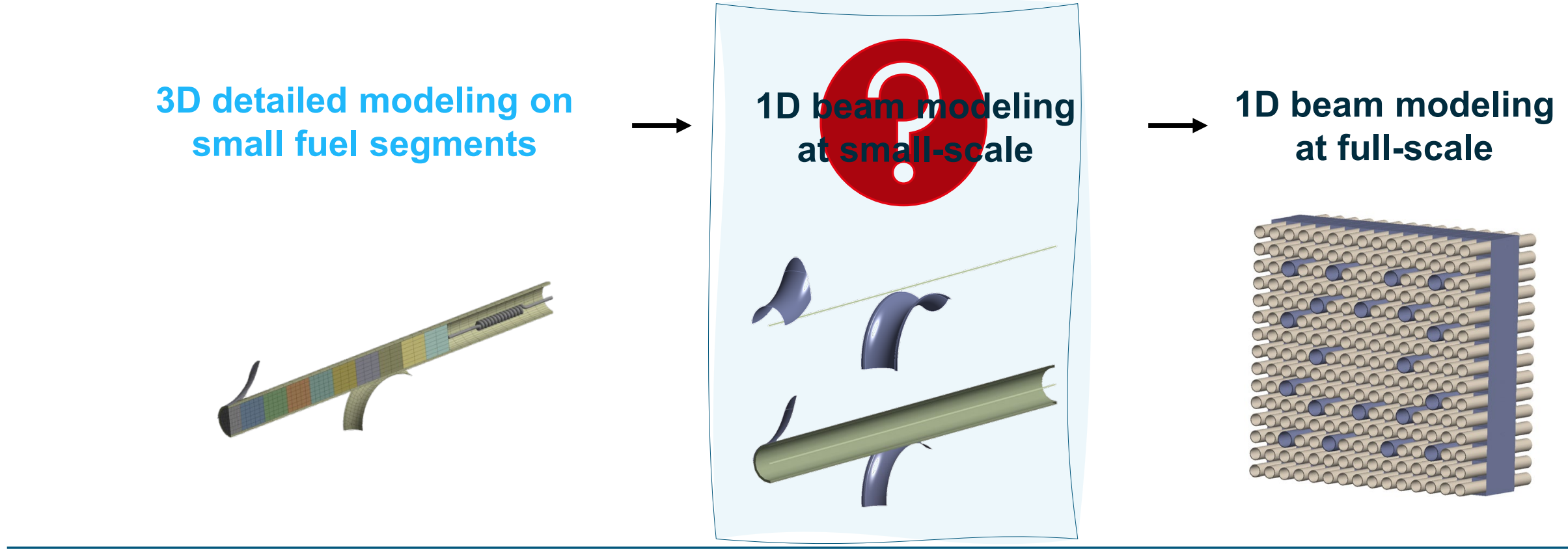

Towards beam modelling for static Thent nuclear fue 


\section{Towards Full-Scale Model - Single Spacer Grid Models}

- Step-wise development of full-scale PWR SFA model

- Spacer grid, rods and guide tubes modeling (from $2 \times 2$ to $5 \times 5$ and $15 \times 15$ )

- Fuel rods modeled using beam elements

- Spacer grid modeled using either beam or shell elements

- ANSYS spring elements are used to model spacer grid springs and dimples

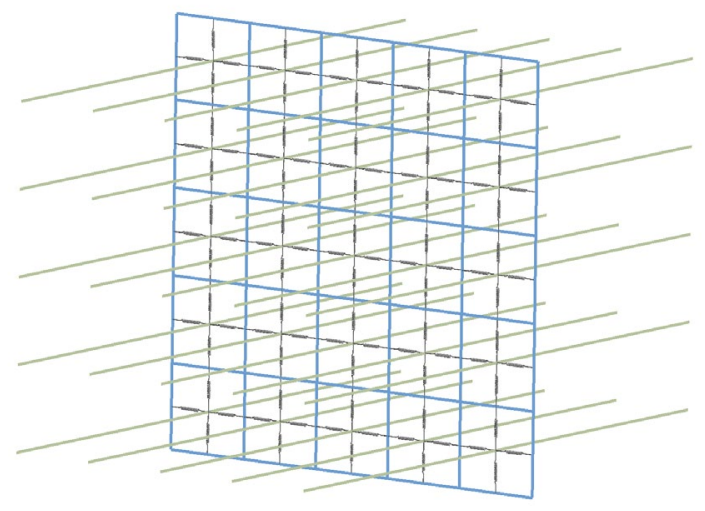

- In each step the model convergence was optimized

- Contacts/interactions between beam-to-beam and beam-to-spacer grid

- Faster convergence
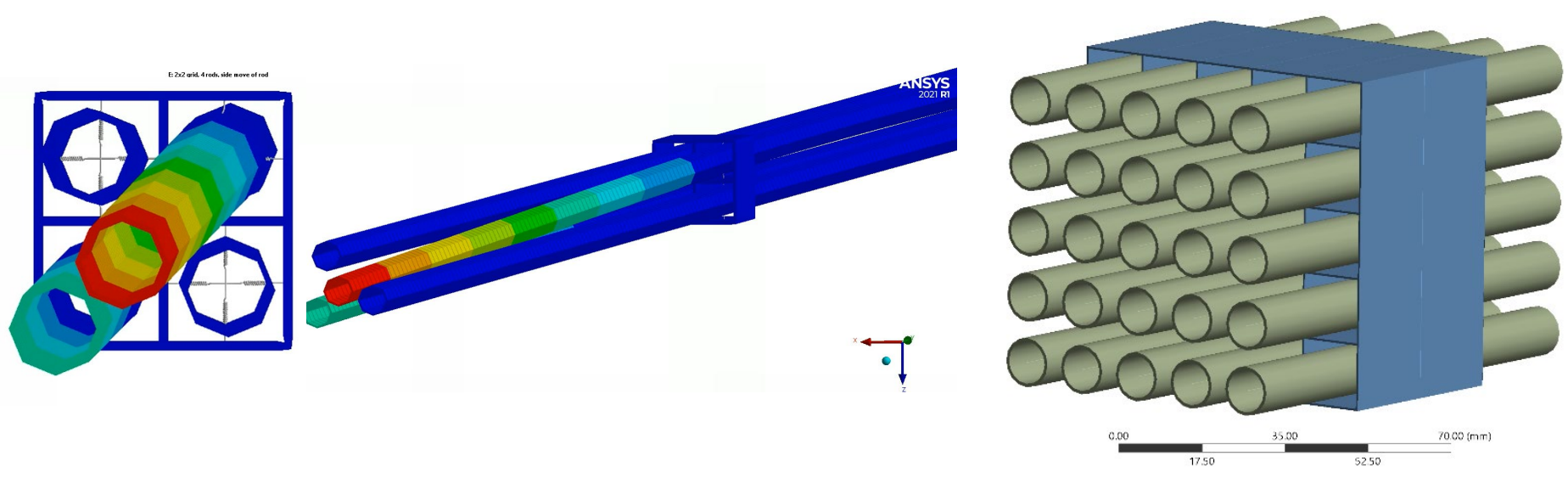

No guide tubes implemented

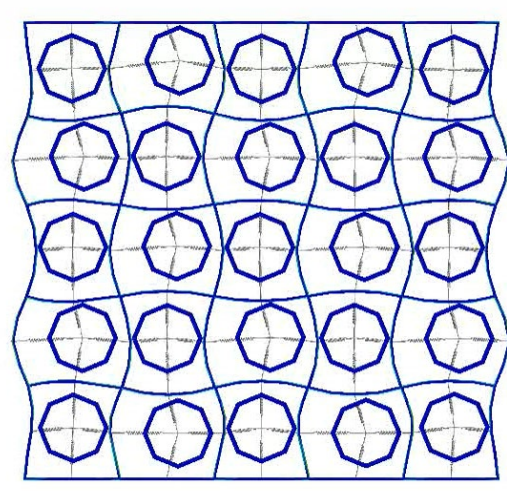

1 guide tube implemente

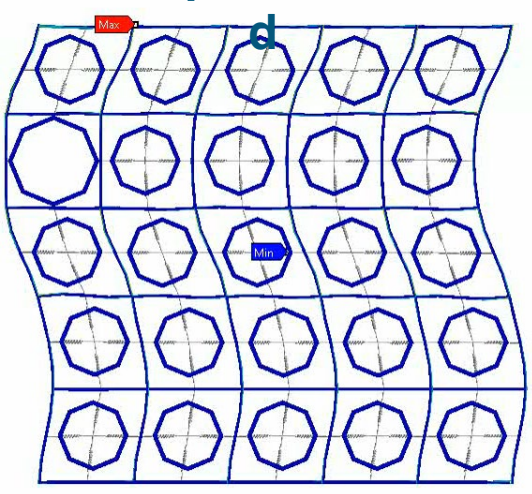




\section{Towards Full-Scale Model - SFA Sub-Model}

- Extension of sub-model to include 4 spacer grids in $15 \times 15$ grid

- Top and bottom end pieces (simplified design - perforated shell element plates)

- Simulation of various loading configurations

- Used to determine model stability and evaluate simulation time
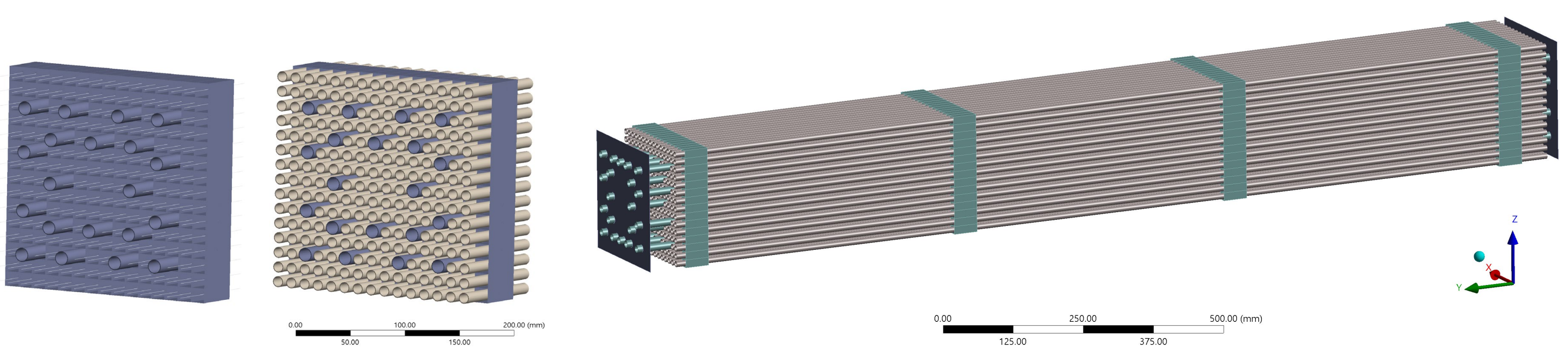


\section{Loading Scenarios Tested}

- Two different loading conditions have been tested simulating postulated accident scenarios:

1. Spacer grid deformation

2. Bending of SFA

SG deformation:

$12 \mathrm{~mm}$ compressive load

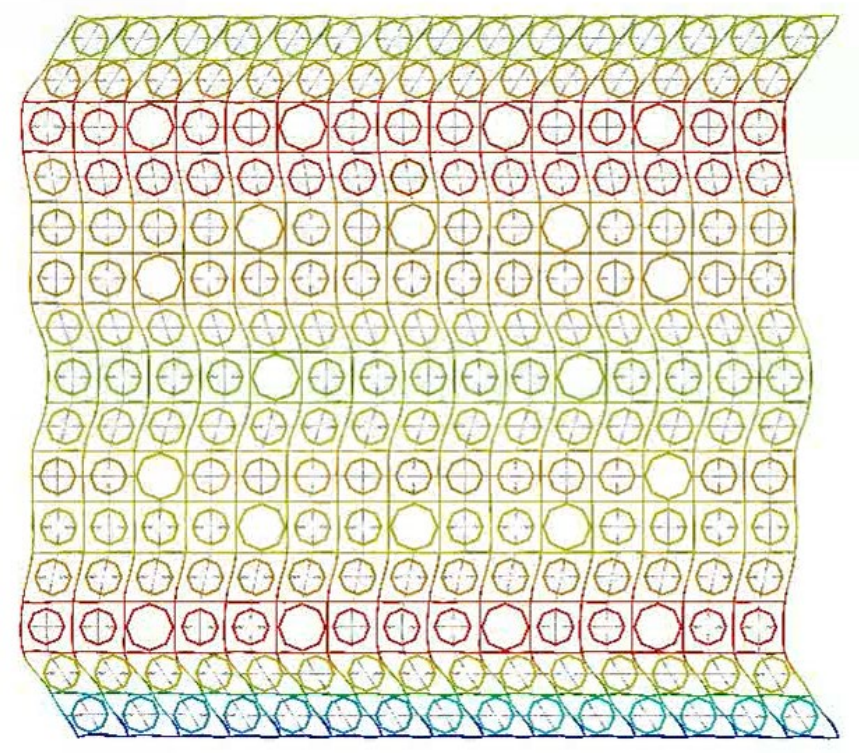

Bending:

Obstacle btw $1^{\text {st }}$ and $2^{\text {nd }}$ spacer grids
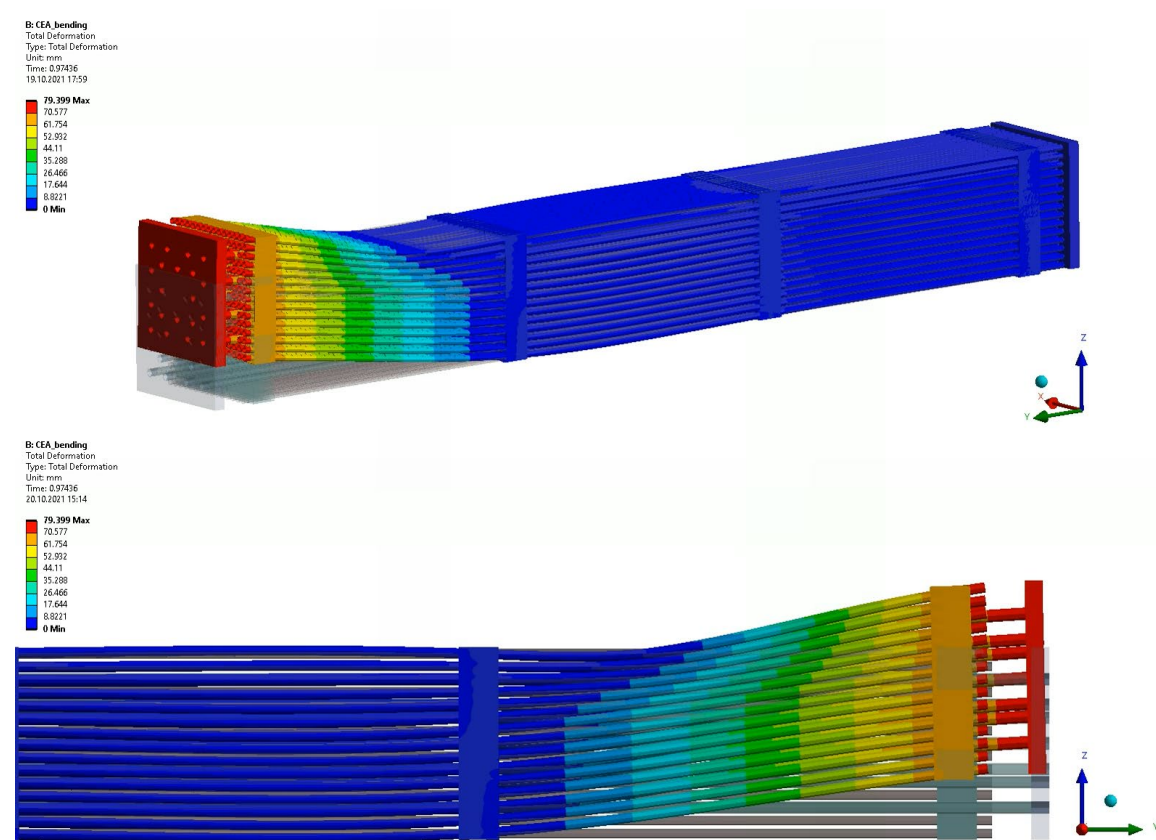


\section{Future Goals}

- Development of full-scale 15x15 PWR SFA model

- Mechanical properties variation based on axial positions

- Burnup profile

- Failure criteria based on rods plastic strain

- Derived from calibration process against experimental results

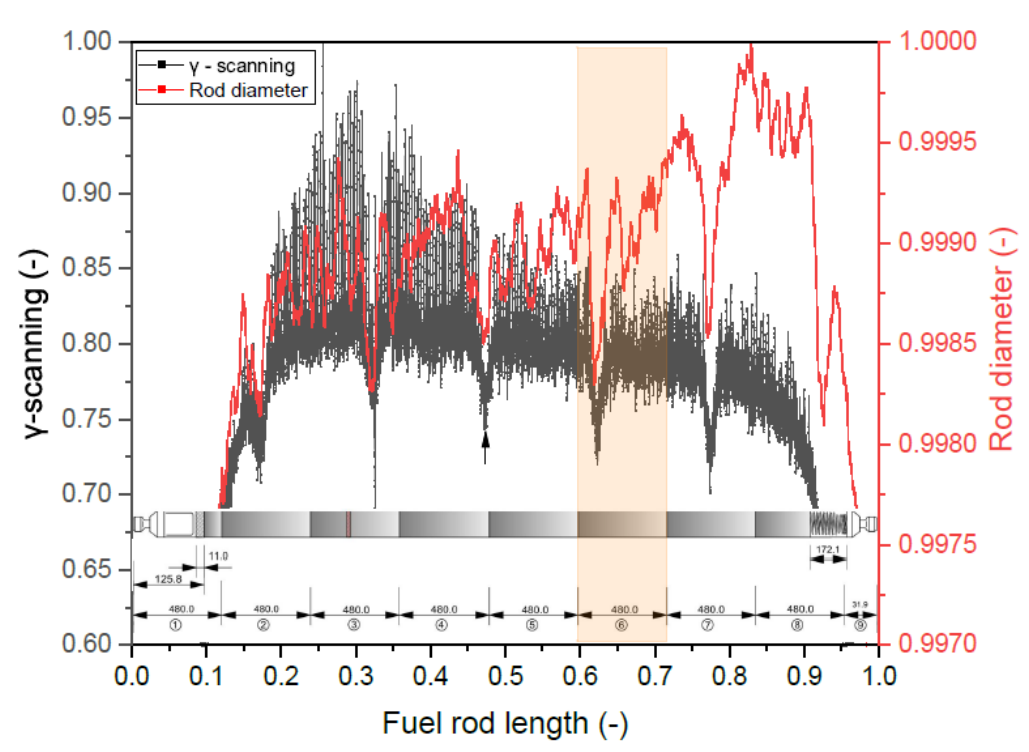

- Investigation of SFA structural integrity under different loading scenarios

- Current data can be used for static analysis under bending loads

- Further optimization of existing and future models to reduce simulation time 
